\title{
The participative arts for people living with a dementia: a critical review
}

\author{
By HANNAH ZEILIG ${ }^{1}$, JOHN KILLICK ${ }^{2}$ \& ChRIS FOX ${ }^{3}$
}

\begin{abstract}
In the last decade, interest has increased in the role of the participative arts for people who are living with a dementia. The flourishing of this area can be partly understood because of an awareness of the potential for art to deliver health care outcomes. In addition, there is widespread agreement that non-pharmacological interventions are important for people living with a dementia. Therefore, participative arts activities have attracted attention as representing beneficial interventions. This critical review which involved the careful mining of academic and grey literature using replicable search strategies contextualises the participatory arts for people living with a dementia and provides an overview of some of the art forms that are most widely used. The review also highlights some of the extant gaps in the knowledge base. The focus is on the UK context but the role of the participative arts for those with a dementia is equally relevant to practitioners in Europe and the US, and therefore some attention was also given to international literature.
\end{abstract}

Keywords: participatory arts, dementia, intervention, health, well-being, quality of life.

${ }^{1}$ Hannah Zeilig, London College of Fashion, University of the Arts, London, UK

${ }^{2}$ John Killick, Dementia Positive, Hebden Bridge, UK

${ }^{3}$ Chris Fox, Norwich Medical School, University of East Anglia, Norwich, UK 
International Journal of Ageing and Later Life

\section{Introduction}

Dementia is a broad term and relates to a group of syndromes rather than one single disease entity. To date, more than 200 subtypes of dementia have been defined and each one has a different pathway or process (Stephan \& Brayne 2010). However, in general terms the dementia syndrome is characterised by progressive decline in cognition of sufficient severity to interfere with activities of daily living (Knopman et al. 2001). The main subtypes include Alzheimer's disease (AD), vascular dementia, dementia with Lewy Bodies, frontal lobe dementia, Pick's disease, and alcohol related dementia (Stephan \& Brayne 2010).

"Dementia" is therefore a syndrome that defies any simple definition; it has become a fear-laden term that encapsulates society's worst terrors (Zeilig 2014a). Despite the general lack of consensus about what dementia is in neurological terms, there is agreement that dementia is a long-term medical disability. To this end, there are regular reports in the media and elsewhere concerning the prevalence of the condition both in Europe and globally. The recent G8 summit (December 2013, London) highlighted the importance of countries working together to find a cure: http:// dementiachallenge.dh.gov.uk/category/g8-dementia-summit/

However, at the present time, finding a cure or even effective drug treatments is proving elusive. Thus a growing body of research and work has steadily focussed on improving quality of life (QOL) ${ }^{1}$ for people living with a dementia (PWD) (Gross et al. 2013). The importance of reducing the use of antipsychotics for PWD and therefore investigating "nonpharmacologic tools" has also been widely embraced across the US (Kaldy 2013). In the absence of appropriate pharmacological interventions the social conditions in which those with a dementia live also need urgent attention. Pioneering work by researchers and practitioners has contributed to the understanding that although Alzheimer's and other dementias may be incurable at present, they are conditions that can be treated and

\footnotetext{
${ }^{1}$ There are debates and disagreements in the research literature concerning 1) how to measure QOL and 2) what QOL actually refers to. The lack of consensus about this contributes to a lack of reliable assessments of projects. It is not within the scope of this review to address these controversies.
} 
The participative arts for people living with a dementia

that treatment always includes more than drugs (Killick 2013; Kitwood 1997; Sabat 2001; Zeisel 2009).

Interest in the social conditions that affect dementia is part of the sociopolitical context that has led to a burgeoning of arts and cultural initiatives for PWD. These initiatives are diverse and reflect an increasingly prevalent assumption that the arts and culture play an intrinsically positive role in health and well-being. However, there has been very little critical evaluation or review of these initiatives and interventions. Indeed, there are gaps in the evidence base relating to the real and measurable benefits from cultural activities for people with a dementia. This is largely because the field is still in its infancy.

The overall aim of the following review is to strengthen the knowledge base concerning the efficacy of arts based approaches for PWD and to investigate the question: What is the value of arts and culture for people living with a dementia? The broad question being explored here is in contrast to previous reviews, which have tended to focus on the specific impact of particular arts interventions on PWD for purposes of evaluation or in order to measure particular variables such as episodic memory or aesthetic stability.

The review is a comprehensive analytical and rigorous discussion. However, it is not systematic as it is not an exhaustive summary, did not use meta-analysis, and although selection bias was minimised by ensuring that colleagues used the same search terms and databases, it was solely the Principal investigator (PI) that was responsible for synthesising the data.

Key databases were searched using the following terms:

(dementia* OR alzheimers OR vascular OR multi-infarct*)

AND

(cultural OR portraiture OR film* OR television OR tv OR memoir* OR novel ${ }^{*}$

OR poe* OR theatre OR performance OR art* OR storytelling OR stories OR music OR sing* OR danc* OR galler* OR archive* OR museum OR "creative writing" OR fashion OR drawing OR paint* OR craft* OR choral OR choir OR panto* OR collage OR design)

AND

UK OR "united kingdom" OR engl* OR welsh OR wales OR Scot* OR Irish or Ireland 
International Journal of Ageing and Later Life

The databases that were used include:

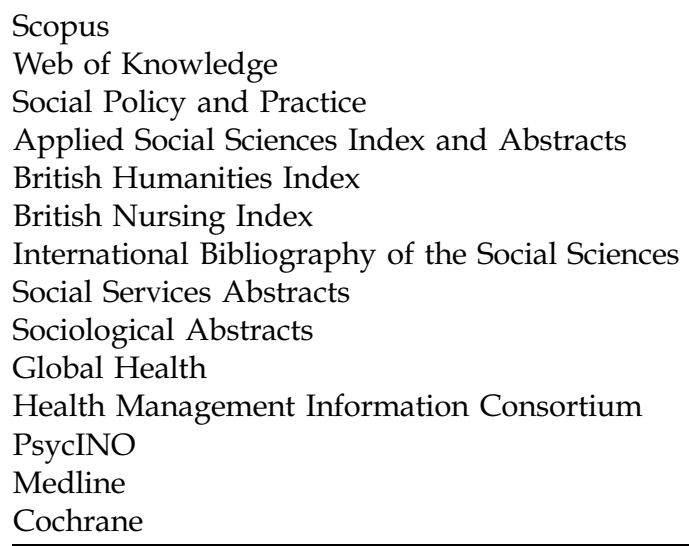

An endnote library was created of 222 references. Of these the articles that were targeted were those including:

- Explicit reference to creative activities that involved older PWD,

- The role of museums and/or art galleries for PWD,

- Participatory arts activities that aim to enhance well-being or QOL for PWD,

- Participatory arts activities that focussed on aesthetic appreciation for PWD,

- Activities that did not include therapies of any kind, ${ }^{2}$

- Systematic data/meta-analyses about the value of the arts for older people, including one Cochrane Review on music therapy for PWD.

A total of 63 articles form the basis for the ensuing examination of the role of the arts for PWD, however the preceding contextual background on the relationship of the arts to health, includes additional references and is provided as an essential framework for the review.

\footnotetext{
2 The focus was on the participatory arts and not therapies (art, music, or drama); however, several articles exploring music as a therapeutic intervention were reviewed where these included relevant methodological information.
} 
The participative arts for people living with a dementia

The evidence pertaining to the role of particular arts activities is then discussed in some detail.

\title{
Context: The Arts and Health
}

\author{
In order to correctly define art, it is necessary, first of all, to cease to consider it as a \\ means to pleasure, and to consider it as one of the conditions of human life. (from: \\ "What Is Art?" by Leo Tolstoy)
}

Debates concerning the relationship of the arts to society can be traced back to Plato and Aristotle and continue to vex contemporary commentators. Tolstoy's conception of art as integral to human life is an argument that has some contemporary resonance. In the twenty-first century in the United Kingdom as beleaguered arts and cultural organisations are facing significant economic challenges (Clift et al. 2009; Mowlah et al. 2014) there is an increasing urge to articulate the pivotal role that the arts and culture play in all aspects of our lives, including centrally to our health and wellbeing. The need to raise awareness concerning the role of the arts as a "strategic national resource" is explicitly stated in a recent report by Arts Council England (Mowlah et al. 2014) and similarly underlies the report by Renshaw (2013). Thus the sociopolitical context in the UK for investigating the value of the participative arts for PWD has also been conditioned by the need for arts organisations to respond to major political changes and funding constraints. This has resulted in an awareness that arts organisations need to actively engage with a more diverse population and a growing appreciation that participatory work/practice that is socially engaged is inherently valuable (Parkinson et al. 2013; Renshaw 2013).

Beyond the important contextual background is the growing body of scientific evidence in support of the value of the arts for health (Clift et al. 2009). Research has evidenced that a higher frequency of engagement with arts and culture is associated with a higher level of subjective well-being (Mowlah et al. 2014) and also that there are instrumental benefits from the arts that can positively affect physical health (Clift 2012). To this end, it has been argued that by supplementing medicine and social care, participation 
International Journal of Ageing and Later Life

in the arts and access to a range of arts opportunities can dramatically improve QOL (Burnham et al. 2007; Cayton 2007; Owen 1999; Staricoff 2006). In connection with this, there has been a growing awareness that health may involve more than simply the absence of disease and that art and aesthetics have an important role to play in delivering health care (Moss \& O'Neill 2014) as well as a reappraisal of the associations between the arts and society in general.

Indeed, the role of the arts for addressing health issues and as a mode for the delivery of social care has been a recurrent topic this century both in Europe and the US (Buttrick et al. 2013; Cohen et al. 2006, 2007; Hanna \& Perlstein 2008; Ramsey 2013). This interest is fuelled by an expanding body of research into the links between culture and flourishing (2013). In addition, there has been explicit interest in the possibility that the arts represent a relatively cost-effective way of alleviating health issues (Ramsey 2013). Work by Cohen (2009) convincingly reports on the cost effectiveness of art programmes in relation to medication usage and visits to the doctor amongst a sample of older people in the US. Of course, successful participatory arts projects are of much greater value to the individuals that take part than the economic benefits they may represent for health or other agencies.

However, it should also be noted that the arts in health movement has not been without its critics. As Clift et al. (2009) have highlighted, a collection of essays by the Mirza (2006) challenged the evidence base for claims concerning the personal and social benefits of the arts. More recently the "fulsome" language and "somewhat uncritical stance" of "evangelists of the arts and health movement" has been criticised:

Equally, reviews of the efficacy of arts in health care ... rarely stand up to sustained academic scrutiny, with a tendency to mould weak data and speculative associations into an often less than convincing polemic. (Moss \& O'Neill 2014: 1032)

It is hoped that as Moss and O'Neill propose, this article will help to contribute to the development of a more reflective and critical line of reasoning concerning the role and value of the arts in particular for PWD. 
The participative arts for people living with a dementia

\section{The Participative Arts for PWD}

Here, the participative arts refer to professional artists that conduct creative or performing arts projects in community settings with PWD and their carers. The purpose of these projects is predominantly to promote health and well-being and is therefore therapeutic. However, it should be noted that in some cases the use of the arts with PWD might also be primarily for aesthetic purposes - in order to create a high quality work of art. Clearly, the therapeutic and aesthetic objectives of a project may overlap and complement one another.

This is distinct from the work of art therapists who typically work in clinical settings (hospitals or hospices) and are usually health care professionals who aim to ameliorate specific conditions (Castora-Binkley et al. 2010). In addition, therapists (whether these are dance/movement, music, drama, or art therapists) tend to focus on the condition (whether this is a dementia, a psychological, or physical problem for instance) and how this can be "treated." For therapists, the arts (whether these are music, painting, theatre) are used as tools to achieve measurable ends. Although this is a valuable approach, the focus of this review is on the arts as a possible means of engaging PWD rather than ways of treating the symptoms or aetiology of the illness itself.

The growth in understanding about residual creative ability that exists in PWD has added to interest in researching and initiating arts projects for PWD (Moriarty 2003; McFadden et al. 2008). Indeed, some practitioners argue that "the peculiar circumstances" of dementia might even release an innate creativity that is connected with an inventive immediacy (Craig \& Killick 2011). Whether or not creativity is augmented by dementia (and at the present time there is scant research on this issue), it is widely appreciated that there is a need to find meaningful and engaging interventions for people who live with a dementia, whether they are living in the community or in care homes, and that the participative arts can improve PWD's moods, enhance their relationships, reduce social isolation, and provide sensory stimulation (Guzmán-García et al. 2013). Moreover, the participative arts may provide unique access to the emotional and physical memories of people who live with a dementia even while their cognitive capacities are diminishing (Eekelaar et al. 2012; McLean 2011; Smith et al. 2012). 
International Journal of Ageing and Later Life

In the past decade there has been an increasing number of arts and cultural initiatives for people who live with a dementia both in the UK and the US (Killick 2012; Roe et al. 2014; Zeilig 2014b; Zeisel 2009). These initiatives are diverse and include music and drama groups, creative writing programmes, dance groups, puppetry, painting classes, and visits to art galleries. Many projects use a range of arts activities to engage participants with a dementia. In the UK there are also a number of organisations that have emerged in the past decade that have a specific focus on using the arts with PWD. In recent years, a growing body of evidence has been compiled exploring the potential of the arts to have an impact on the health and wellbeing of PWD (Basting 2009; Camic et al. 2013b; Fritsch et al. 2009; Ledger \& Edwards 2011). However, this field is nascent - and consolidating evidence about the varied and often small scale, short-term projects, is problematic.

As is cogently noted by McLean (2011) in her evidence review of the impact of the participatory arts on older people, this is a new and emerging research field, with little available high quality research. This is echoed by Roe and colleagues (2014) in their discussion of the "creative arts" for PWD:

A strong evidence base to inform such creative arts activities is lacking ... Rigorous evaluation and research evidence is required to support their continuing development. (4)

Similarly, there has been scant work exploring the views of PWD concerning their perceptions of the value of arts and culturally based activities.

\section{Singing and Music Groups}

The only Cochrane review of the arts for PWD that has been located examines the evidence relating to music therapy (Vink et al. 2006) (updated in 2010) and is therefore not directly relevant here. However it is significant that of all possible art practices it is music that has been examined in a Cochrane review. This perhaps indicates that questions regarding the therapeutic value of music for PWD have a particular interest for investigators and practitioners alike. The specific focus of this 
The participative arts for people living with a dementia

review was to assess whether music therapy can diminish behavioural and cognitive problems or improve social and emotional functioning. Ten studies were included that used a range of behavioural and psychological tools as outcome measures. However, the overall conclusion by Vink et al. (2006) was that the methodological quality and the reporting of the included studies were too poor to draw any useful conclusions. Sherratt et al. (2004) similarly highlight the limitations of research studies in this area.

Weaknesses in methods and questions about the reliability of data are equally pertinent when considering the role of other participative arts considered in this review. Many studies are beset with methodological difficulties and are unable to formulate robust conclusions. Nevertheless, there have been several convincing and methodologically stringent $\mathrm{RCTs}^{3}$ investigating the role of music and singing for PWD (Cooke et al. 2010; Harrison et al. 2010; Särkämö et al. 2014). These studies indicate the benefits of music and singing for PWD; albeit with varying emphases.

Harrison et al. (2010) and Cooke et al. (2010) report on different aspects of the same randomised controlled trial using a cross-over design - with a music intervention and a reading control group. This RCT studied the effects of a live music programme on agitation, emotion, and QOL for PWD. Standardised, quantitative measures were used including the Dementia Quality of Life and Geriatric Depression Scales. Qualitative data was also gathered but solely from the musicians involved in the programme. Although the reflective data from the musicians indicated that the intervention protocol was successful in terms of promoting improvements in mood, memory, general well-being, and QOL for PWD; the standardised measures used with PWD did not provide evidence that the therapeutic use of live music was more effective than a group reading activity. However, results did suggest that both the music and reading activities offered

\footnotetext{
${ }^{3} \mathrm{RCT}$ refers to a randomised controlled trial. This is the method used in evidence based clinical trials whereby a group of patients is randomised into an experimental group and a control group. Using randomisation is one way in which any possibility of selection bias in a trial is minimised.
} 
International Journal of Ageing and Later Life

opportunities to improve sense of belonging, self-esteem, and depressive symptoms in some PWD.

Särkämö et al. (2014) similarly explored QOL for PWD. This study adopted a single blind RCT approach to determine the long-term efficacy of a novel dyadic music intervention on PWD. A comprehensive neuropsychological testing battery was used to evaluate cognitive domains (Särkämö et al. 2014) and standardised scales including the Cornell-Brown Scale for QOL were used to evaluate mood. This careful systematic approach to the possible effects of music on cognitive, emotional, and social aspects of life for a group of PWD with mild/moderate dementia and their family members demonstrates that everyday musical leisure activities can have an important role in maintaining cognitive ability, enhancing mood and QOL, and promoting the well-being of family members. The study also indicates the potential for using RCT designs when investigating longerterm effects of arts activities.

Definitions of music vary (Spiro 2010) and may include "active" improvised musical activities or activities that include music but do not necessarily have it as a main focus. Music listening amongst PWD has also been investigated. This is primarily a receptive activity and represents a passive form of musical interaction. The effects of playing customised music on the levels of agitation and depression amongst PWD was studied by Janata (2012). The study was designed as a controlled, randomised, singlesite trial lasting 16 weeks. Weekly data samples were collected for the Neuropsychiatric Inventory, the Cohen-Mansfield Agitation Inventory, and the Cornell Scale for Depression in Dementia (Janata 2012).

This study was inconclusive and it is hypothesised that this is because of the particular assessments used in this study (Janata 2012). The authors conclude that more detailed examination of the interaction between music, specific agitation behaviours, dementia severity, and time of day is warranted.

Thus there is a developing literature on the benefits of music for PWD in relation to the behavioural symptoms of dementia and sense of well-being (Gerdner 2000; Hara 2011; Rose 2008; van der Geer et al. 2009). In addition, the specific ability of music to allay cognitive decline based on understandings generated by the neuroscience of music has also been studied (Särkämö et al. 2012, 2014). These latter studies indicate that music may be 
The participative arts for people living with a dementia

able to reduce emotional and behavioural disturbances and have positive effects on cognitive functioning. Yet, methodological "pitfalls" (Särkämö et al. 2012) mean that conclusions about the long-term benefits and practical applicability of music interventions remain tenuous.

The value of singing in dementia care has also been widely noted (Götell et al. 2009). This has been reinforced by the "Singing for the Brain" model and Silver Song Club Project (Skingley \& Vella-Burrows 2010). The evaluation by Bannan and Montgomery-Smith (2008) demonstrated that singing was an enjoyable activity for PWD and also had the potential to enhance QOL and well-being for their carers. The role of singing for improving health has been established for other cohorts in wide ranging studies (Cohen et al. 2006, 2007; Clift et al. 2009).

Camic et al. also observe the intense enjoyment for PWD derived from singing (Camic et al. 2013b). This was a small scale but thorough study (10 PWD and carers over a 10-week period) (Camic et al. 2013b) that used quantitative and qualitative evaluative measures. The objective of the study was to assess whether engagement in singing groups positively affected PWD. Standardised measures included assessing the PWD's cognitive abilities at baseline with the Addenbrooke Cognitive Examination and subsequently at post-group and 10-week follow up. Their mood was assessed with the Geriatric Depression Scale and QOL with the Dementia Quality of Life (Dem-QOL-proxy), behavioural and psychological problems with the Neuropsychiatric Inventory and activities of daily living with the Bristol Activities of Daily Living Scale (Camic et al. 2013b). In addition to these standardised measures, levels of engagement and participation were measured using a "specially constructed" observational scale (Camic et al. 2013b). The qualitative data provided a different perspective than the quantitative data; whilst the former demonstrated the enthusiasm with which PWD participated, the standardised measures were inconclusive. Indeed, the benefit of using qualitative data with statistical analysis is also appreciated in the RCT study of a music intervention for PWD (Harrison et al. 2010).

The study by Camic et al. did not support the commonly held belief that PWD are not interested or capable of taking on new activities (Camic et al. 2013b). Singing and music have also been used with people who are in more advanced stages of dementia and have lost all verbal skills or who 
International Journal of Ageing and Later Life

are completely bedridden (Povey 2011), however more rigorous studies about the role of music and singing for PWD who are in more advanced stages of the illness are required.

\section{Art Groups: Drawing, Making and Painting}

Although there may be numerous projects that use art with PWD, research into art-making activities for PWD is sparse (Eekelaar et al. 2012). However, despite small samples there is evidence that those with a dementia who engage in arts activities experience improvements in social and psychological well-being. For example, Kinney and Rentz (2005) used a careful method of evaluation to compare the effectiveness of a drawing and painting programme (Memories in the Making) led by an artist facilitator with participation in a structured activity on the well-being of twelve adults with a dementia at 2 day centres. Well-being was assessed using an outcomes-based observation tool designed specifically for this study and that focussed on seven domains of well-being. This study reported that participants demonstrated increased sustained attention, engagement, and communication. However, these findings are tentative because of the small sample size and certain aspects of the observation tool developed (Kinney \& Rentz 2005).

The work of the Magic Me group (http://www.magicme.co.uk) has also been successful at engaging PWD with the creation of artworks (Gilfoy \& Knocker 2009) as has the small scale "Lighting Up" project (Sherratt 2010). However, both accounts of artists working with PWD would benefit from both a theoretical framework and from longer-term evaluation that assesses the duration of the benefits experienced by PWD who participate. As noted by Salisbury et al. (2011), partial explanation for the benefits of arts interventions for PWD comes from neurological findings that demonstrate how drawing therapeutically affects the brain. Future research in this area might benefit from incorporating and investigating these findings.

Evidence from recent studies on aesthetic perception amongst those living with a dementia as noted by Graham et al. (2013), suggests that interactions with art can lessen the severity of $\mathrm{AD}$ symptoms. The interesting results of Graham et al.'s work (2013) indicate that: 
The participative arts for people living with a dementia

... people with AD do possess aesthetic perception much like that of healthy adults, we must explain how this is possible given that brain areas that seem to underlie normal aesthetic perception appear to be damaged in AD. (6)

The authors speculate that apparent damage may not be so severe and that sub-cortical systems could help individuals compensate. If aesthetic judgments remain stable then the value of interventions involving art viewing are evident. This is a finding that is echoed in earlier studies (Halpern et al. 2008). Clearly a deeper understanding about the role of visual information may also lead to the development of novel therapies and programmes. It may even be that other areas of aesthetic judgment also remain stable.

Eekelaar et al. (2012) in a project that gathered data in novel ways including audio recordings investigated the association between visual arts based interventions (in the context of an art gallery) and cognitive stimulation for PWD (Eekelaar et al. 2012). The study design involved measuring cognitive responses to a semi-structured interview by PWD and audio recording these, as an alternative to standardised questionnaires, which have proved problematic in research with PWD. Because of the research focus that was on PWD's episodic memory and verbal fluency, questions about the gallery's artwork were used to elicit verbal responses (Eekelaar et al. 2012). The study procedure (once consent had been obtained) was for the PWD and carer to attend three 90-minute gallery sessions over 3 weeks. The final hour of the session was spent in an artmaking session. Four weeks post intervention, participants were interviewed again, shown reproductions of the art they had viewed, and asked for feedback about the group. This study, although limited in scope and scale - six PWD participated and the study took place over 7 weeks without a control group - indicated that episodic memory and verbal fluency could be enhanced both by art viewing and art-making. This is a finding that deserves further investigation.

Ullán et al. (2013) designed a monochrome photographic procedure for people living with a "mild or moderate" form of dementia. Above all, this project demonstrates that the PWD who participated were able to use new technologies and learn new skills. Participants also enjoyed the activity. However, this study was highly selective (those with more advanced forms 
International Journal of Ageing and Later Life

of dementia were not included) and only provides information about the process of the workshops rather than their longer-term impact.

\section{Dance Projects}

Since the beginning of the twenty-first century there has been a marked escalation in projects and approaches using dance with PWD. In the UK the work of Richard Coaten has been central to fostering understanding about the intrinsic value of movement, dance, and the imagination for PWD (Coaten 2002).

Dancing with PWD has been explored in hospital settings and day centres and positive effects have been noted. These include a decrease in challenging behaviour and the promotion of person-centred care (Coaten et al. 2013). In addition, there has been increasing recognition of the benefits of non-verbal, body-oriented focus for PWD and the embodied nature of the illness, in contrast to the predominant emphasis in western culture on verbal, cognitively-based activities (Coaten \& Newman-Bluestein 2013; Kontos 2005). Dance projects can increase movement range and mobility in PWD and therefore nurture remaining capacities (Coaten et al. 2013; Smith et al. 2012). Dance is inherently interactive and playful and these are important qualities that dance projects can offer PWD, their carers, and the staff of day centres/care homes (Coaten \& Newman-Bluestein 2013; Smith et al. 2012). However, a recent systematic review of dance as a psychosocial intervention in care homes (Guzmán-García et al. 2013) highlights that evidence on the efficacy of dancing in care homes is limited because of the methodological challenges facing this research.

The "Moving Memories" project (Coaten et al. 2013) was relatively long term (36 weeks) and involved a group of 10-14 PWD as well as care workers, a researcher, and the dance artist who led the activity. The project explored the role that movement can play in the lives of those who live with a dementia and also for care staff within a day centre. The report stresses the value of having an independent researcher who was able to use ethnographic processes (such as diaries) to capture the spirit of dance. Similarly, the pilot project, led by Smith et al. (2012), used qualitative methods to gather data about the dance intervention. However, in their reflective report, Smith et al. (2012) comment upon the limitations of relying upon feedback interviews with participants and the need for future 
The participative arts for people living with a dementia

evaluations to include film and visual mechanisms especially in dance projects.

\section{Theatre and Storytelling}

A primary benefit of drama-based projects is the opportunity to express emotions and thus cope with the condition of living with a dementia (Harries et al. 2013). After all, telling stories and acting these out is a fundamental human experience and remains a key method for sharing experiences.

Participatory theatre activities that include creative storytelling for PWD have been pioneered in the US by Anne Basting in her innovative Time Slips project (2001). Basting's "Finding Penelope" project (a site specific performance based in a Milwaukee care home; Mello \& Voights 2012) has demonstrated that PWD are able to learn new skills and actively take part, even co-direct a theatrical production. The evaluation of "Finding Penelope" was qualitative and drew on a wide variety of sources including: video recordings of meetings, in-depth interviews, and photographs amongst other data (Mello \& Voights 2012). The goal of this project was to transform long-term care through creative engagement ... and also to change the conditions of care for persons with dementia. As eloquently noted by Zeisel (2009) the dramatic ambience itself conveys feelings and ideas and thus drama provides a powerful mode of communication for PWD. This was evidenced by Basting's project, which was also important in generating a sense of belonging amongst participants (Zeisel 2012). The evaluation of "Finding Penelope" whilst arguably limited (it was written by a member of the project team) found that the theatrical project had made a positive difference in the quality of residents' and staff lives.

Basting's work is important in encouraging creative work that does not rely on memory. The Storybox project (a UK theatre based project) similarly emphasises creating rather than reminiscing, this is associated with a failure-free atmosphere (Harries et al. 2013). In addition, improvisation is often central to participatory theatre. Improvisation allows PWD to dare, to play, and to create, and is a process that many find liberating (Basting 2001). Improvisation also facilitates inclusion by fostering collaboration, fun, and experimentation (Harries et al. 2013). The model of interactive theatre that has been used with PWD by the group "Ladder 
International Journal of Ageing and Later Life

to the Moon" (Zeisel 2009) similarly encourages co-creation and experimentation as a means of involving as many PWD as possible.

The dramatic staging and performing of the stories of PWD is an important means of combatting prejudice and stigma about dementia (Zeisel 2009). Even within care homes, theatrical performances have had demonstrably positive effects on the attitudes of staff - as outlined in the evaluative report of the "Ladder to the Moon" residency (Parsons 2009). However, whether altered attitudes are evidenced in changed caring practices is less apparent (Parsons 2009).

\section{Poetry and Writing}

Poetry appeals to similar parts of the brain as song (Zeisel 2009). The need for rhythm and rhyme is innately human and it is posited that this is one of the reasons that working with PWD to create poetry is so appropriate. In the UK John Killick has pioneered ways of working with PWD to co-write poetry. This work and the poems produced (Killick 1997, 2007, 2008) have contributed to insights into the worlds of some individuals living with a dementia.

In addition, Killick's work has persistently emphasised the existence of selfhood, the ability to communicate in language, and to exercise creative choice even at relatively late stages of a dementia. The importance of the collective creation in creating poetry with PWD is also highlighted in other studies (Aadlandsvik 2008). Participants might not be able to write any longer but can direct the poet (who acts as an amanuensis for the PWD) and this allows PWD some degree of control and autonomy. The one-to-one interactions of a poet with a PWD and the personal nature of the activity may be a major factor contributing to the success of these interventions (Gregory 2011).

Poetry is also used with PWD as something to recite together or to read with PWD and has been found to be a means of releasing an individual's personality (Glazner 2006). Creating poetry as a means of encouraging reminiscence was the focus of the "Try to Remember" project (2011); however the views and benefits for PWD were not the explicit focus of this study. A study of a literature-based intervention for PWD (Billington et al. 2013) that addressed the impact and effect of shared reading on PWD found that there was a significant reduction in dementia symptom 
The participative arts for people living with a dementia

severity but similarly failed to investigate the views or opinions of PWD in any detail.

Despite the anecdotal evidence that many projects use poetry and creative writing with PWD, studies evaluating the efficacy of these interventions (especially from the point of view of the PWD) are scant and work examining the applications of poetry using rigorous, robust methodologies is rather sparse.

\section{The Role of Museums and Art Galleries}

Museums are public places that are non-stigmatising and that have websites that extend beyond the boundaries of the physical place (Camic \& Chatterjee 2013). However, despite the increase in understanding about the possible cognitive and psychological benefits of arts and health interventions in museums, research is still at an early stage (Camic \& Chatterjee 2013).

Art galleries as a "normal," intellectually stimulating location for social interaction have great potential for engaging PWD especially given the possibilities that aesthetic perception remains relatively stable for PWD (Graham et al. 2013). Art galleries can use their resources to devise projects that engage PWD quite easily (Camic \& Chatterjee 2013; Camic 2013a; MacPherson et al. 2009; Rosenberg et al. 2009; Zeisel 2009). Participatory projects in art galleries can use a variety of methods for engagement, including structured discussions of actual artworks followed by opportunities for art-making (Camic et al. 2013a; Roe et al. 2014). Indeed the controversial area of new learning for PWD has been confronted by Camic both in the context of a singing group (2013), and within a gallery setting (2013a). In both instances, the research team found that PWD were able to undertake new learning.

Traditional art gallery settings have been the focus for most studies exploring the influence of the location of participatory arts projects for PWD (Camic \& Chatterjee 2013; Camic et al. 2013a; Eekelaar et al. 2012; MacPherson et al. 2009; Rosenberg et al. 2009). This area could be expanded by exploring the effects of similar projects at different, less conventional types of galleries and museums (as noted by Camic et al. 2013a).

Memories that are visually encoded are vivid and can be easily stimulated; this explains the powerful nature of visual stimulation in art 
International Journal of Ageing and Later Life

galleries, as noted by Rosenberg et al. (2009). Similarly, Zeisel (2009) makes numerous observations about the possibilities that PWD have for aesthetic appreciation of art that are based on his experience of museum tours with his "Artists for Alzheimer's" programme.

\section{Issues of Evaluation}

The thorny issue of adequately evaluating complex interventions such as participative arts projects: that involve interrelated variables, confounding factors, and a range of possible outcomes, was a recurring theme in the literature reviewed. Some of the main concerns relate to the use of inappropriate outcome measures; for example, Camic et al. (2013b) highlight the inadequacy of standardised QOL measures for people with a dementia. In addition, overtly clinical approaches to evaluating the participative arts for example, investigating cognitive effects or the impact of "doses" of music - has also been queried (Harries et al. 2013; de Medeiros \& Basting 2014).

However, it is important to note that it is not solely the participative arts for PWD that are difficult to evaluate. In their general guidance on the evaluation of complex interventions, the Medical Research Council notes that whilst there are some aspects of good practice that are evident:

... on many important issues there is no consensus yet on what is best practice. (Craig et al. 2008: 6)

In large part, this is because of the methodological and practical constraints that affect the evaluation of complex interventions.

\section{Discussion}

According to the literature reviewed, the participative arts are able to contribute positively to the lives of those living with a dementia in manifold ways. These include: aiding communication; encouraging residual creative abilities; promoting new learning; enhancing cognitive function; increasing confidence, self-esteem, and social participation; and generating a sense of freedom - among other documented benefits. In addition, the arts are effective at alleviating boundaries between service providers and people with dementia and in providing new insights for the dementia workforce and family members. The arts have a unique application for uncovering 
The participative arts for people living with a dementia

and communicating the interior worlds of those living with a dementia (Gjengedal et al. 2014; Ryan et al. 2009). The spate of recent theatre productions, memoirs, and films exploring various aspects of living with a dementia indicate that there is a receptive and wide audience for artistic contemplations of this condition.

Yet the complex role of the participative arts for PWD is a field that is in its infancy. Moreover, because of methodological challenges, and a dearth of theoretically informed work, most conclusions are tentative rather than confident, particularly concerning the long-term impact of arts projects. However, recent studies using RCT designs to investigate singing and music for PWD (Harrison et al. 2010; Särkämö et al. 2014) reflect the growing interest in systematic research in this area. As noted elsewhere:

The basic research has begun, and the field is now ready for a deeper exploration .... (Spiro 2010: 897)

In concurrence, this review indicates that the evidence base can certainly be strengthened. In particular, studies that take place over longer time periods with more diverse groups of PWD and that use a variety of evaluative methods (several studies have demonstrated the efficacy of combining qualitative with quantitative measures) are needed.

As Hara points out, in particular relation to research on the effects of music on PWD Hara (2011), the neuroscientific approach tends to examine music as an isolated phenomenon (separate from the social context) and to examine it solely in terms of its ability to effect change. Thus the effect of music is measured in a specific and predefined way, much like a pharmaceutical drug. This aptly highlights a key difficulty in evaluating the role of the participative arts for PWD - the number of external, confounding factors, and the importance of appreciating the whole context. Studies that explore the underlying cognitive, neurological, and emotional mechanisms that may be affected are required. In addition, studies that draw on appropriate theoretical models and that consciously integrate the views of participants living with a dementia should be conducted in order to make reliable conclusions that are generally applicable and have practical relevance for PWD. Furthermore, economic analysis should be included in studies of the participatory arts for PWD so that commissioning agencies can make decisions regarding the relative expense of these 
International Journal of Ageing and Later Life

groups (Camic et al. 2013b) in comparison with other therapeutic or pharmacological interventions.

Despite the need for further stringent evaluative research, the work that has already been conducted indicates that the participative arts will continue to develop and respond to the needs of PWD in uniquely valuable ways. In particular, recent work in the UK (Zeilig et al. 2014), indicates that feelings of peace may be generated for PWD by music making, and that arts projects can produce internal connections and also friendships. In addition, arts projects can create a space that is outside the demands of daily life and the ability for PWD to engage reciprocally. Whilst it is difficult to establish whether these effects are intrinsically associated with the arts activity as opposed to any other form of social participation; the inherent emphasis of most arts projects is on group creation and playfulness. The focus on play and creation privileges the present moment and is particularly valuable for PWD.

After all, the emphasis of the arts is on experience, feeling, imagination, and meanings that lie to the side of our vision, meanings that may be masked and cannot easily be measured. The emphasis on emotions that is integral to the arts is of particular relevance for PWD and their carers. Moreover, if health is more than the absence of disease and is primarily about adaptation, understanding, and acceptance (Davies et al. 2014) then the arts may indeed be more powerful for those living with a dementia than anything medicine has to offer.

\section{Acknowledgements}

This literature review forms essential background for Mark Making a study exploring the role and value of the participative arts for PWD. This was funded by the Arts and Humanities Research Council and supported by the University of the Arts, London. The research team is indebted to the artists, participants, and staff of the participative arts projects that they worked with.

\section{Corresponding Author}

Hannah Zeilig, London College of Fashion, University of the Arts, 20 John Prince's Street, London W1G 0BJ, UK. Email: h.zeilig@fashion.arts.ac.uk 
The participative arts for people living with a dementia

\section{References}

Aadlandsvik, R. (2008). The second sight: Learning about and with dementia by means of poetry. Dementia 7(3): 321-339.

Bannan, N. \& Montgomery-Smith, C. (2008). Singing for the brain: Reflections on the human capacity for music arising from a pilot study of group singing with Alzheimer's patients. The Journal of the Royal Society for the Promotion of Health 128(2): 73-78.

Basting, A. (2001). "God Is a Talking Horse": Dementia and the performance of self. The Drama Review 45(3): 78-96.

Basting, A. D. (2009). Forget Memory: Creating Better Lives for People with Dementia. Baltimore: John Hopkins University Press.

Billington, J., Carroll, J., Davis, P., Healey, C. \& Kinderman, P. (2013). A literature-based intervention for older people living with dementia. Perspectives in Public Health 133(3): 165-173.

Burnham, A., Lammy, D., Cayton, H. \& Hewitt, P. (2007). A Prospectus for Arts and Health. London: Department of Health.

Buttrick, J., Parkinson, A., Wallis, A., Trentham, S. \& Riseborough, M. (2013). What do we know about the role of arts in the delivery of social care? London: Skills For Care.

Camic, P. M. \& Chatterjee, H. J. (2013). Museums and art galleries as partners for public health interventions. Perspectives in Public Health 133(1): 66-71.

Camic, P. M., Tischler, V. \& Pearman, C. H. (2013a). Viewing and making art together: A multi-session art-gallery-based intervention for people with dementia and their carers. Aging \& Mental Health 18(2): 161-168.

Camic, P. M., Williams, C. M. \& Meeten, F. (2013b). Does a 'Singing Together Group' improve the quality of life of people with a dementia and their carers? A pilot evaluation study. Dementia 12(2): 157-176.

Castora-Binkley, M., Noelker, L., Prohaska, T. \& Satariano, W. (2010). Impact of arts participation on health outcomes for older adults. Journal of Aging, Humanities, and the Arts 4(1): 352-367.

Cayton, H. (2007). Report of the Review of Arts and Health Working Group. London: Department of Health.

Clift, S. (2012). Creative arts as a public health resource: Moving from practice-based research to evidence-based practice. Perspectives in Public Health 132(3): 120-127. 
International Journal of Ageing and Later Life

Clift, S., Camic, P. M., Chapman, B., Clayton, G., Daykin, N., Eades, G., Parkinson, C., Secker, J., Stickley, T. \& White, M. (2009). The state of arts and health in England. Arts \& Health 1(1): 6-35.

Coaten, R. (2002). Movement Matters: Revealing the hidden humanity within dementia through movement, dance and the imagination. Dementia: The International Journal of Social Research and Practice 1(3): 386-391.

Coaten, R., Heeley, T. \& Spitzer, N. (2013). Dancemind 's' moving memories' evaluation and analysis; a UK based dance and health project for people living with dementia and their care-staff. UNESCO Observatory Multi-Disciplinary Journal in the Arts 3(3): 1-17.

Coaten, R. \& Newman-Bluestein, D. (2013). Embodiment and dementia Dance movement psychotherapists respond. Dementia 12(6): 677-681.

Cohen, G. D. (2009). New theories and research findings on the positive influence of music and art on health with ageing. Arts $\mathcal{E}$ Health: An International Journal for Research, Policy and Practice 1(11): 48-62.

Cohen, G. D., Perlstein, S., Chapline, J., Kelly, J., Firth, K. M. \& Simmens, S. (2006). The impact of professionally conducted cultural programs on the physical health, mental health, and social functioning of older adults. The Gerontologist 46(6): 726-734.

Cohen, G. D., Perlstein, S., Chapline, J., Kelly, J., Firth, K. M. \& Simmens, S. (2007). The impact of professionally conducted cultural programs on the physical health, mental health, and social functioning of older Adults - 2-Year results. Journal of Aging, Humanities, and the Arts 1(1-2): 5-22.

Cooke, M., Moyle, W., Harrison, S. \& Murfield, J. (2010). A randomized controlled trial exploring the effect of music on quality of life and depression in older people with dementia. Journal of Health Psychology 15(5): 765-776.

Craig, C. \& Killick, J. (2011). Why creativity and dementia go together. Journal of Dementia Care 19(6): 20-22.

Craig, P., Dieppe, P., Macintyre, S., Michie, S., Nazareth, I. \& Petticrew, M. (2008). Developing and Evaluating Complex Interventions: New Guidance. London: Medical Research Council.

Davies, C. R., Knuiman, M. \& Wright, P. (2014). The art of being healthy: A qualitative study to develop a thematic framework for understanding 
The participative arts for people living with a dementia

the relationship between health and arts. British Medical Journal Open 4: 1-10. doi: 10.1136/ bmjopen-2014-004790.

de Medeiros, K. \& Basting, A. (2014). "Shall I compare thee to a dose of Donepezil?": Cultural arts interventions in dementia care research. The Gerontologist 54(3): 344-353.

Eekelaar, C., Camic, P. M. \& Springham, N. (2012). Art galleries, episodic memory and verbal fluency in dementia: An exploratory study. Psychology of Aesthetics, Creativity, and the Arts 6(3): 262-272.

Fritsch, T., Kwak, J., Grant, S., Lang, J., Montgomery, R. R. \& Basting, A. D. (2009). Impact of TimeSlips, a creative expression intervention program, on nursing home residents with dementia and their caregivers. The Gerontologist 49(1): 117-127.

Gerdner, L. A. (2000). Music, art, and recreational therapies in the treatment of behavioral and psychological symptoms of dementia. International Psychogeriatrics 12(Suppl S1): 359-366.

Gilfoy, K. \& Knocker, S. (2009). Magic across the generations. Journal of Dementia Care 17(3): 22-25.

Gjengedal, E., Lykkeslet, E., Sørbø, J. I. \& Sæther, W. H. (2014). Brightness in dark places': Theatre as an arena for communicating life with dementia. Dementia 13(5): 598-612.

Glazner, G. (2006). The rhyme and reason of poetry therapy. Care Advantage (Spring): 24-27.

Götell, E., Brown, S. \& Ekman, S. (2009). The influence of caregiver singing and background music on vocally expressed emotions and moods in dementia care: A qualitative analysis. International Journal of Nursing Studies 46(4): 422-430.

Graham, D. J., Stockinger, S. \& Leder, H. (2013). An island of stability: Art images and natural scenes - but not natural faces - show consistent esthetic response in Alzheimer's-related dementia. Frontiers in Psychology 4(107): 1-8.

Gregory, H. (2011). Using poetry to improve the quality of life and care for people with dementia: A qualitative analysis of the Try to Remember programme. Arts \& Health 3(2): 160-172.

Gross, S. M., Danilova, D., Vandehey, M. A. \& Diekhoff, G. M. (2013). Creativity and dementia: Does artistic activity affect well-being beyond the art class? Dementia doi: 10.1177/1471301213488899. 
International Journal of Ageing and Later Life

Guzmán-García, A., Hughes, J. C., James, I. A. \& Rochester, L. (2013). Dancing as a psychosocial intervention in care homes: A systematic review of the literature. International Journal of Geriatric Psychiatry 28(9): 914-924.

Halpern, A. R., Ly, J., Elkin-Frankston, S. \& O'Connor, M. G. (2008). 'know what I like': Stability of aesthetic preference in Alzheimer's patients. Brain and Cognition 66: 65-72.

Hanna, G. \& Perlstein, S. (2008). Creativity Matters: Arts and Aging in America. Washington, DC: National Center for Creative Aging, Monograph.

Hara, M. (2011). Music in dementia care: Increased understanding through mixed research methods. Music $\mathcal{E}$ Arts in Action 3(2): 34-58.

Harries, B., Keady, J. \& Swarbrick, C. (2013). The Storybox Project: Examining the Role of a Theatre and Arts-Based Intervention for People with Dementia. Manchester: University of Manchester.

Harrison, S., Cooke, M., Moyle, W., Shum, D. \& Murfield, J. (2010). Delivering a music intervention in a randomized controlled trial involving older people with dementia: Musician experiences and reflections. Music and Medicine 2(4): 214-218.

Janata, P. (2012). Effects of widespread and frequent personalized music programming on agitation and depression in assisted living facility residents with Alzheimer-Type dementia. Music and Medicine 4(1): 8-15.

Kaldy, J. (2013). Alternative therapies for a new era of health care. The Consultant Pharmacist 28(2): 84-90.

Killick, J. (1997). You Are Words. London: Hawker.

Killick, J. (2007). Dementia and 'Me-ness'. The poetry of Ian McQueen. Alzheimer's Care Quarterly 8(1): 8-11.

Killick, J. (2008). It moves you, it hits you inside. Reading poems at Redholme. Journal of Dementia Care 16(6): 28-29.

Killick, J. (2012). Working as an Artist with People with Dementia, Briefing Report for National Association of Writers in Education (NAWE). Available on http://www.nawe.co.uk/writing-in-education/writingand-community/artworks/nawe-lab.html

Killick, J. (2013). Dementia Positive. Edinburgh: Luath Press.

Kinney, J. M. \& Rentz, C. A. (2005). Observed well-being among individuals with dementia: Memories in the Making ${ }^{\mathbb{C}}$, an art program, 
versus other structured activity. American Journal of Alzheimer's Disease and Other Dementias 20(4): 220-227.

Kitwood, T. (1997). Dementia Reconsidered, the Person Comes First. Maidenhead: Open University Press.

Knopman, D., DeKosky, S. T., Cummings, J., Chui, H., Corey-Bloom, J. \& Stevens, J. (2001). Practice parameter: Diagnosis of dementia: An evidence-based review. Report of the quality standards committee of the American Academy of Neurology. Neurology 56(9): 1143-1153.

Kontos, P. C. (2005). Embodied selfhood in Alzheimer's disease: Rethinking person-centred care. Dementia 4(4): 553-570.

Ledger, A. \& Edwards, J. (2011). Arts-based research practices in music therapy research: Existing and potential developments. The Arts in Psychotherapy 38(5): 312-317.

MacPherson, S., Bird, M., Anderson, K., Davis, T. \& Blair, A. (2009). An art gallery access programme for people with dementia: You do it for the moment. Aging \& Mental Health 13(5): 744-752.

McFadden, S. H., Frank, V. \& Dysert, A. (2008). Creativity in the "Now" of advanced dementia: Glimpses of the lifeworld through storytelling and painting. Journal of Aging, Humanities, and the Arts 2(2): 135-149.

McLean, J. (2011). An Evidence Review of the Impact of Participatory Arts on Older People. London: Mental Health Foundation.

Mello, R. \& Voights, J. (2012). The Penelope Project: Using the Power of Myth to Transform Long Term Care. Available on http:// www.thepenelopeproject. com/links/materials/penelope-program-evaluation (Accessed: March 18, 2014).

Mirza, M. (ed.) (2006). Culture Vultures: Is UK arts policy damaging the arts? London: Policy Exchange.

Moriarty, J. (2003). Innovative practice. Dementia 2(1): 125-135.

Moss, H. \& O'Neill, D. (2014). The art of medicine: Aesthetic deprivation in clinical settings. The Lancet 383(9922): 1032-1033.

Mowlah, A., Niblett, V., Blackburn, J. \& Harris, M. (2014). The Value of Arts and Culture to People and Society. Manchester: Arts Council England.

Owen, J. W. (1999). Arts, health and wellbeing: A third way for health? World Hospitals and Health Service: The Official Journal of the International Hospital Federation 35(2): 3-6. 
International Journal of Ageing and Later Life

Parkinson, A., Buttrick, J., Wallis, A., Trentham, S. \& Riseborough, M. (2013). What Do we Know about the Role of Arts in the Delivery of Social Care? London: Skills for Care.

Parsons, M. (2009). Over the Moon: Effectiveness of Using Interactive Drama in a Dementia Care Setting, Evaluation Report. London: London Centre for Dementia Care.

Povey, S. (2011). Music lessons: Listening with your whole being. Journal of Dementia Care 19(4): 32-33.

Ramsey, P. (2013). Arts and older people strategy 2010-2013, Arts Council of Northern Ireland. Cultural Trends 22(3-4): 270-277.

Renshaw. (2013). Being in Tune, A Provocation Paper: Seeking Ways of Addressing Isolation and Dislocation Through Engaging in the Arts. London: Guildhall School of Music and Drama.

Roe, B., McCormick, S., Lucas, T., Gallagher, W., Winn, A. \& Elkin, S. (2014). Coffee, cake \& culture: Evaluation of an art for health programme for older people in the community. Dementia. doi: 10.1177/ 1471301214528927. Available on http://dem.sagepub.com/content/ early/2014/03/25/1471301214528927 (Accessed: April 14, 2014).

Rose, L. (2008). Music for Life: A model for reflective practice. Journal of Dementia Care 16(3): 20-23.

Rosenberg, F., Parsa, A., Humble, L. \& McGee, C. (2009). Meet Me: Making Art Accessible to People with Dementia. New York: Museum of Modern Art.

Ryan, E. B., Bannister, K. A. \& Anas, A. P. (2009). The dementia narrative: Writing to reclaim social identity. Journal of Aging Studies 23(3): 145-157.

Sabat, S. R. (2001). The Experience of Alzheimer's Disease. Life Through a Tangled Veil. Oxford: Blackwell.

Salisbury, K., Algar, K. \& Windle, G. (2011). Arts programmes and quality of life for people with dementia: A review. Journal of Dementia Care 19(3): 33.

Särkämö, T., Laitinen, S., Tervaniemi, M., Numminen, A., Kurki, M. \& Rantanen, P. (2012). Music, emotion, and dementia: Insight from neuroscientific and clinical research. Music and Medicine 4(3): 153-162.

Särkämö, T., Tervaniemi, M., Laitinen, S., Numminen, A., Kurki, M., Johnson, J. K. \& Rantanen, P. (2014). Cognitive, emotional, and social 
The participative arts for people living with a dementia

benefits of regular musical activities in early dementia: Randomized controlled study. The Gerontologist 54(4): 634-650.

Sherratt, C. (2010). Lighting up: Lessons learned in establishing an arts project. Journal of Dementia Care 18(6): 22-23.

Sherratt, K., Thornton, A. \& Hatton, C. (2004). Music Interventions for People with Dementia: A Review of the Literature. Aging \& Mental Health 8(1): 3-12.

Skingley, A. \& Vella-Burrows, T. (2010). Therapeutic effects of music and singing for older people. Nursing Standard 24(19): 35-41.

Smith, N., Waller, D., Colvin, A., Naylor, M. \& Hayes, J. (2012). Dance and Dementia Project: Findings from the Pilot Study. Brighton: Unviersity of Brighton.

Spiro, N. (2010). Music and dementia: Observing effects and searching for underlying theories. Aging \& Mental Health 14(8): 891-899.

Staricoff, R. L. (2006). Arts in health: The value of evaluation. Journal of the Royal Society for the Promotion of Health 126(3): 116-120.

Stephan, B. \& Brayne, C. (2010). Prevalence and projections of dementia. In M. Downs \& B. Bowers (eds.), Excellence in Dementia Care: Research into Practice (pp. 9-35). Berkshire: Open University Press.

Ullán, A. M., Belver, M. H., Badía, M., Moreno, C., Garrido, E., Gómez-Isla, J., Gonzalez-Ingelmo, E., Delgado, J., Serrano, I., Herrero, C., Manzanera, P. \& Tejedor, L. (2013). Contributions of an artistic educational program for older people with early dementia: An exploratory qualitative study. Dementia 12(4): 425-446.

van der Geer, E. R., Vink, A. C., Schols, J. M. G. A. \& Slaets, J. P. J. (2009). Music in the nursing home: Hitting the right note! The provision of music to dementia patients with verbal and vocal agitation in Dutch nursing homes. International Psychogeriatrics 21(01): 86-93.

Vink, A. C., Birks, J., Bruinsma, M. S. \& Scholten, R. J. P. M. (2006). Music Therapy for People with dementia. Cochrane Database of Systematic Reviews (3): CD003477. doi: 10.1002/14651858.CD003477.pub2.

Zeilig, H. (2014a). Dementia as a cultural metaphor. The Gerontologist 54(2): 258-267.

Zeilig, H. (2014b). Gaps and spaces: Representations of dementia in contemporary British poetry. Dementia 13(2): 160-175. 
International Journal of Ageing and Later Life

Zeilig, H., Killick, J. \& Fox, C. (2014). Mark Making: A Critical Review of the Value of Arts and Culture for People Living with a Dementia. London: University of the Arts and Humanities Research Council.

Zeisel, J. (2009). I'm Still Here: A Breakthrough Approach to Understanding Someone Living with Alzheimer's. New York: Penguin Group (USA). 